\title{
Reccurrence of GH-secreting pituitary adenomas during puberty in children with germline AIP mutations: a clinical challenge
}

\author{
Filipponi $\mathrm{S}^{1}$, Esposito $\mathrm{V}^{1}$, Daly $\mathrm{AF}^{2}$, Beckers $\mathrm{A}^{2}$, Jaffrain-Rea $\mathrm{ML}^{1,2,3}$

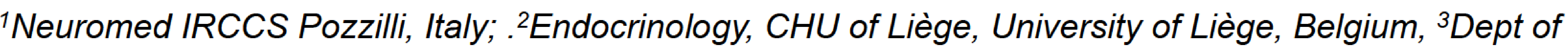 \\ Biotechnological and Applied Clinical Sciences, University of L'Aquila, Italy.
}

Introduction
Germline AIP mutations (AIPmut)
predispose to young onset somatotroph
pituitary adenomas (GHPA) and
gigantism. AIPmut GHPA are often
aggressive and resistant $\quad$ to
pharmacological treatment, which may
be especially challenging in a pediatric
setting. We report our experience with
two young Italian male patients, who
developed incipient gigantism (Fig.1)
due to AIPmut pituitary macroadenomas.
After successful surgery, both
experienced recurrent $\quad$ GH/IGF1
hypersecretion followed by tumour
regrowth during puberty. Diagnostic and
therapeutic challenges are reported.

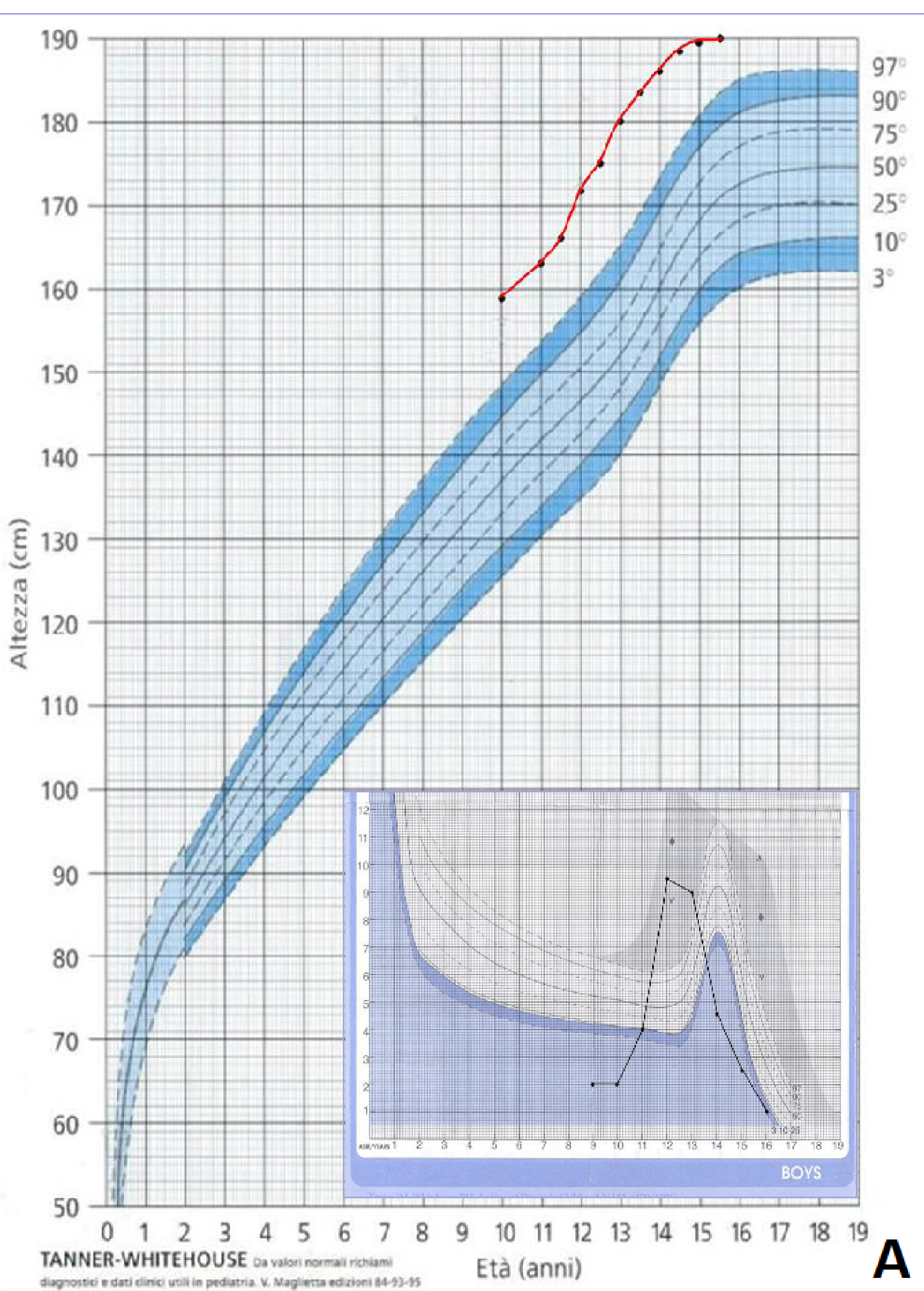

Case 1. Patient $A$ developed incipient gigantism in a familial AIP-related setting $\left(A^{\prime} P^{R 304 X}\right)$ at the age of 8 yrs-old. Two years after successful transphenoidal surgery for an intrasellar macroadenoma (12 mm) (Fig.2) spontaneous puberty occurred and IGF1 increased progressively. Final diagnosis of disease recurrence was made at the age of $14.5 \mathrm{yrs}$ and pharmacological treatment with cabergoline (CAB) and somatostatin analogues (SSA) was started with a partial and transient response, and a recent shift to pegvisomant. A small intrasellar recurrence recently appeared and second surgery is being considered. Height has stabilized at $191 \mathrm{~cm}$.

Case 2. Patient B complained of visual defects at the age of $11.5 \mathrm{yrs}$ in a context of accelerated growth. MRI showed a pituitary macroadenoma with suprasellar extension (27 mm) (Fig.3), which was successfully operated on. Plasma GH/IGF1/PRL and growth velocity normalized. A germline AIPA277P mutation was found (Fig.4). Spontaneous puberty occurred and IGF1 increased progressively. Two years after surgery, he was started on CAB due to modest biochemical/clinical worsening and initial tumour regrowth. The recurrence become increasingly aggressive, requiring multiple treatment modalities with SSA, CAB and/or pegvisomant. He was re-operated at 14 and $18 \mathrm{yr}$-old s and irradiated at 19 (2013). Medical therapy was progressively withdrawn, and GH/IGF1 hypersecretion is currently controlled with a still preserved pituitary function. Final height is $190 \mathrm{~cm}$.

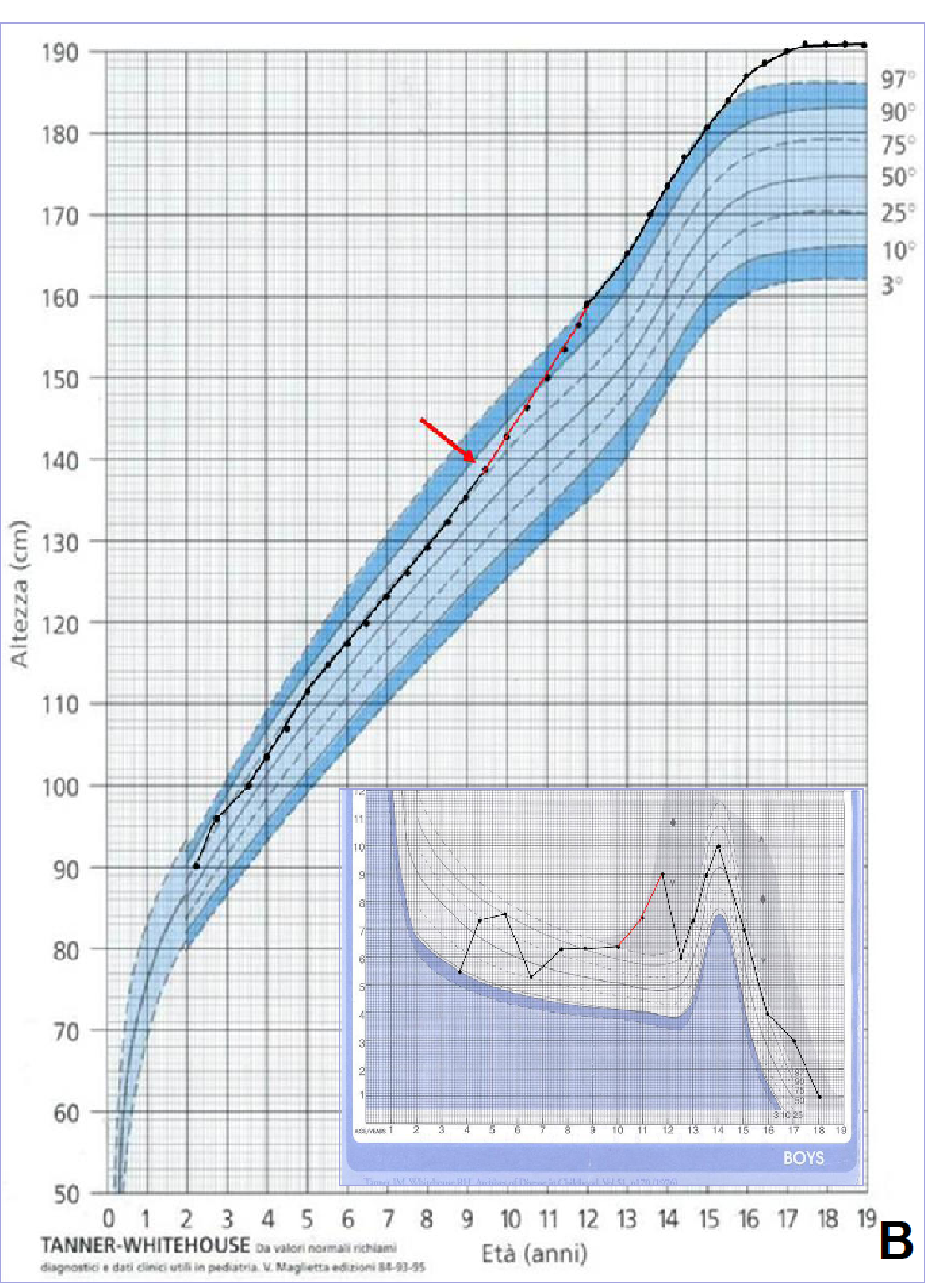

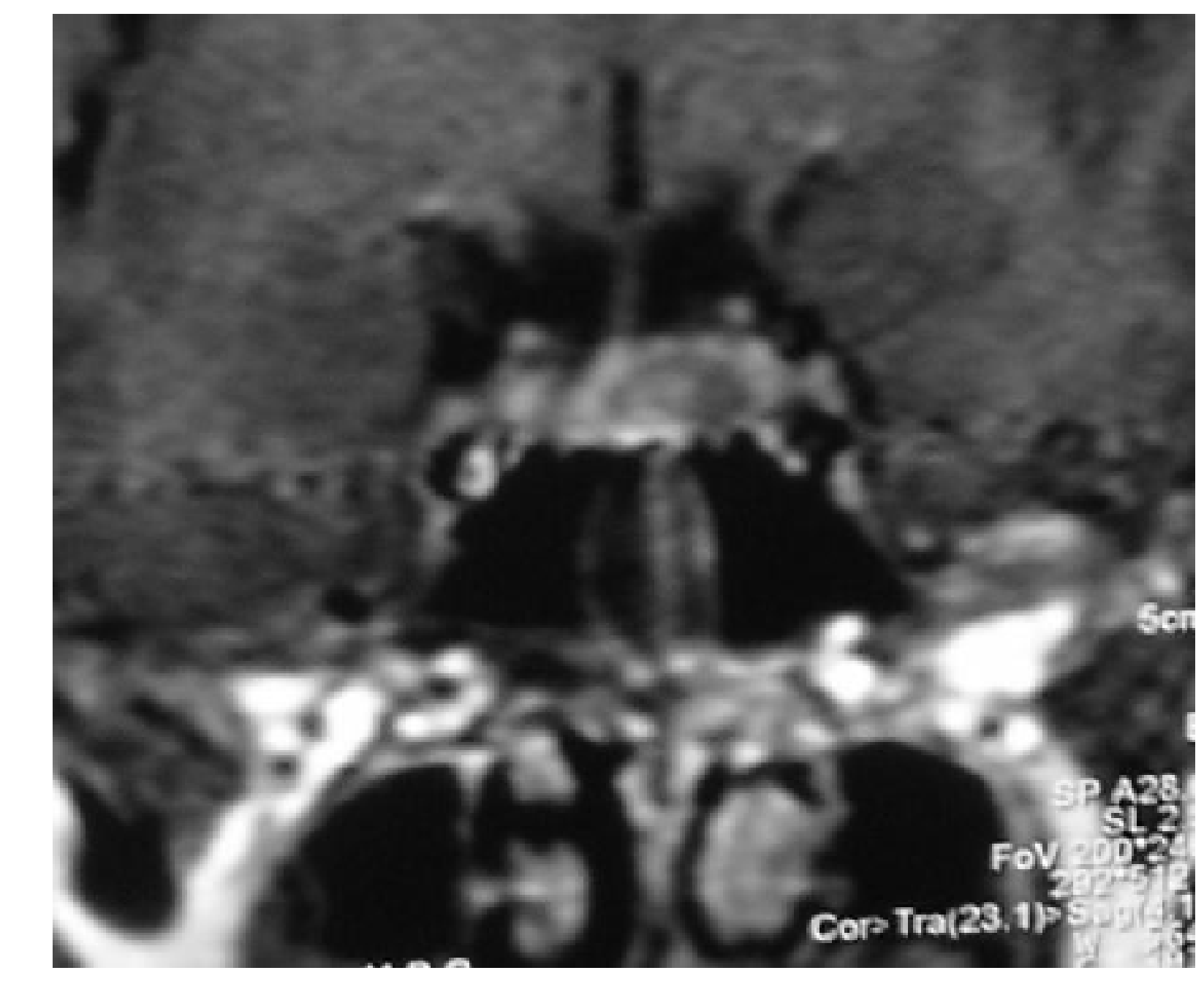

Fig. 2: An intrasellar pituitary macroadenoma (Case 1).

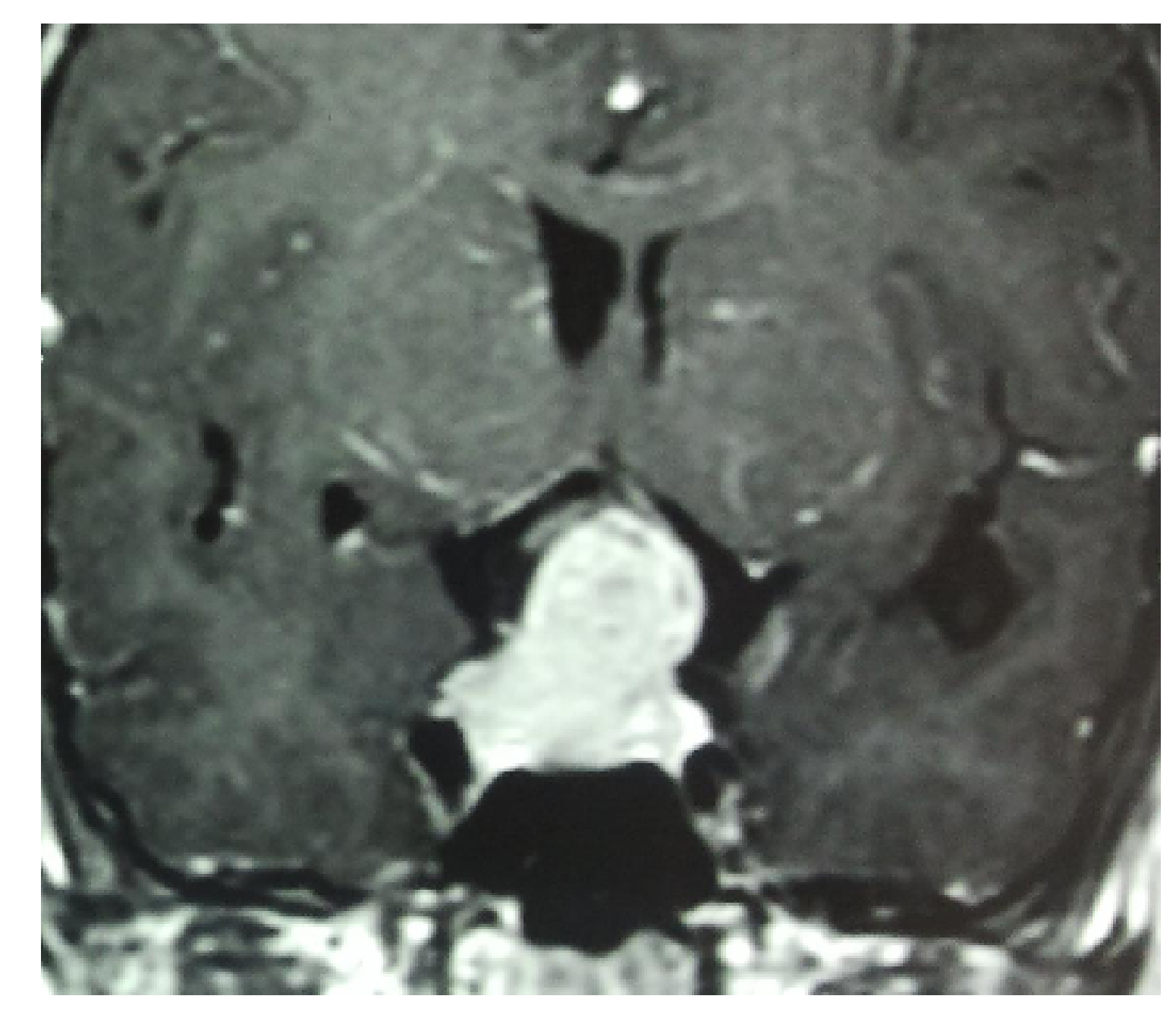

Fig. 3: An invasive pituitary macroadenoma (Case 2).

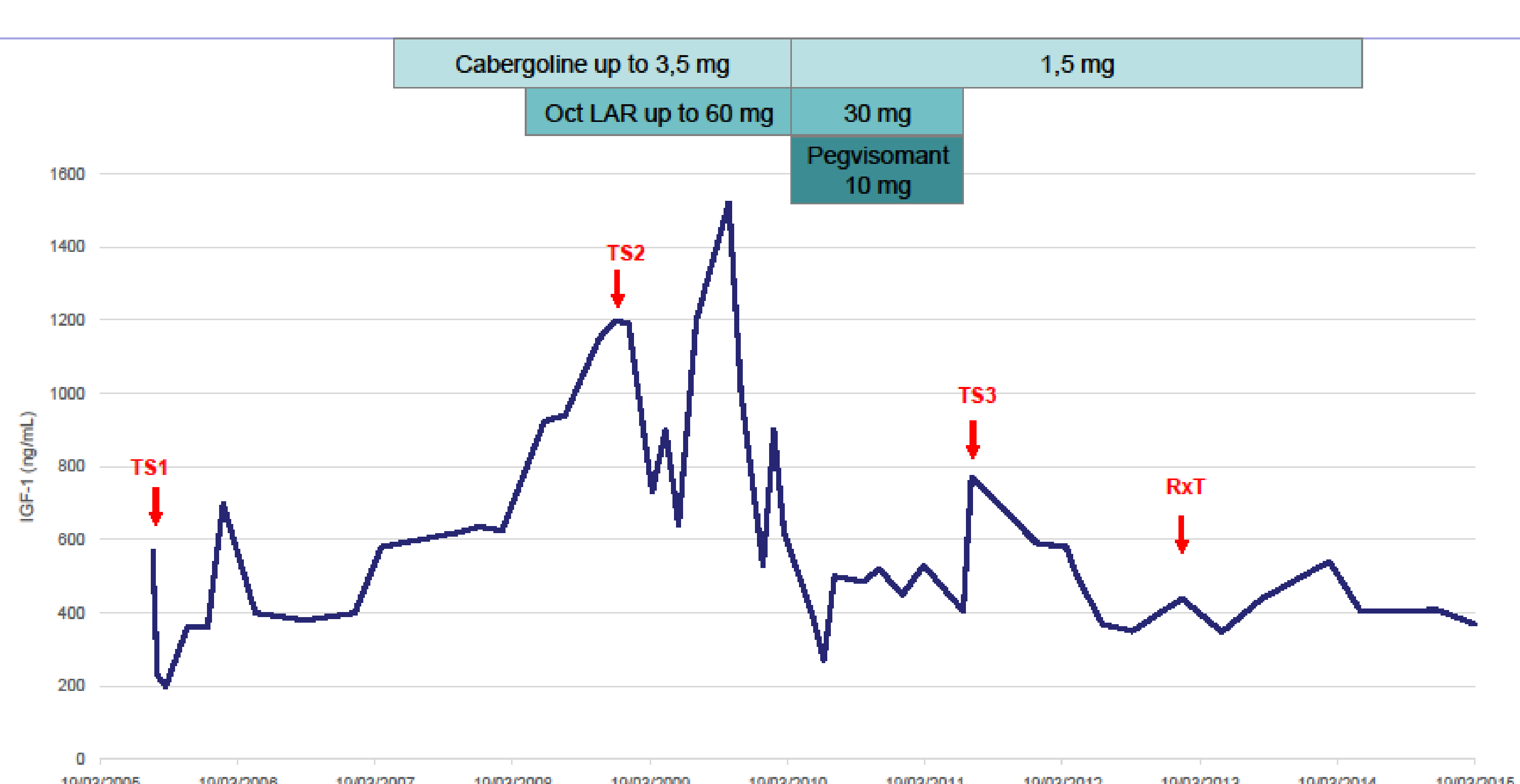

Fig. 1: Tanner growth chart and height velocity in two AIPmut pediatric patients (A: Case 1; B: Case 2)

Fig. 4: IGF-1 values variations in patient 2

Conclusion. While confirming that AIPmut patients may develop pituitary adenomas very early, thereby supporting pre-pubertal screening in AIPmut kindreds, these observations illustrate the peculiarities of peri-pubertal disease management in this setting. Most of the IGF1 levels were beyond the upper normal IGF1 limits for age during puberty, despite active disease, and MRI was of limited help in the early phase of recurrence. In both cases increasing resistance to SSA and CAB occurred, but the IGF1 target on Pegvisomant treatment is ill-defined. Thus, although the early diagnosis and treatment of disease recurrence is of special importance in patients with incipient gigantism, this may be very challenging during puberty. Indications for repeated surgery and/or radiotherapy at a young age should also be balanced with the risk of long-term complications.

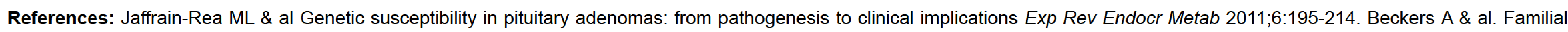
isolated pituitary adenomas (FIPA) and the pituitary adenoma predisposition due to mutations in the AIP gene. Endocr Rev. 2013;34:239-277. 